\title{
THE SEARCH FOR RADIO EMISSION FROM GIANT EXOPLANETS
}

\author{
J.-M. Grießmeier*
}

\begin{abstract}
The intensity of Jupiter's auroral radio emission quickly gave rise to the question whether a comparable coherent emission from the magnetosphere of an extrasolar planet could be detectable. A simple estimation shows that exoplanetary auroral radio emission would have to be at least 1000 times more intense than Jupiter's emission to be detectable with current radio telescopes. Theoretical models suggest that, at least in certain cases, the radio emission of giant exoplanets may indeed reach such an intensity. At the same time, in order to generate such an emission, an exoplanet would need to have a sufficiently strong intrinsic planetary magnetic field. Extrasolar planets are indeed expected to have a magnetic field, but to date, their magnetic field has never been detected. As discussed elsewhere [Grießmeier et al., 2015], the most promising technique to unambiguously observe exoplanetary magnetic fields is to search for the planetary auroral radio emission. The detection of such an emission would thus constitute the first unambiguous detection of an exoplanetary magnetic field. We review recent theoretical studies and discuss their results for the two main parameters, namely the maximum emission frequency and the intensity of the radio emission. The predicted values indicate that detection should be possible using modern low-frequency radio telescopes. We also review past observation attempts, and compare their sensitivity to the predicted emission.
\end{abstract}

\section{Introduction}

In the history of PRE conferences and PRE conference proceedings, this is not the first time magnetospheric radio emission from extrasolar planets have been discussed. Even though observations of this emission have first been attempted four decades ago (see Section 4 for details), i.e. before this conference series even started, one of the seminal papers of this field was published in this line of proceedings [Zarka et al., 1997], and other studies followed (within and outside of the frame of the PRE conferences). So far, no

\footnotetext{
* LPC2E - Université d'Orléans / CNRS, 45071 Orléans cedex 2, France \& Station de Radioastronomie de Nançay, Observatoire de Paris, PSL Research University, CNRS, Univ. Orléans, OSUC, 18330 Nançay, France
} 
firm detection has yet been achieved; still, a lot of progress has been made since the first observation attempts and publications. In particular, a number of search programs have been conducted, and a number of theoretical developments have been made. The aim of the present article is to give an overview over some of these recent developments.

Three other articles in this volume are relevant in the context of magnetospheric radio emission from exoplanets: Turner et al. [2017] present an ongoing observation program with the Low Frequency Array (LOFAR). Zarka et al. [2017] solve the long-standing question whether (exo)planetary radio emission is powered by the kinetic or magnetic energy of the solar wind, and Weber et al. [2017a] study the expanded ionospheres of evaporating hot Jupiter and discuss whether such planets should be the preferred targets for radio observations (which was frequently assumed in the past).

\section{Recent theoretical studies}

Despite the fact that the theoretical aspects of exoplanetary auroral radio emission have been studied extensively for almost twenty years now, there are regularly new studies which come up with surprising results, some of which can have important implications for the target selection for observational studies. Rather than giving a complete overview over all studies that have been performed over the last two decades, we refer the readers to recent reviews [Zarka, 2011; Grießmeier, 2015] and concentrate on the more recent studies that have been performed since these review articles have been published.

\subsection{On exoplanetary magnetic fields}

In analogy to the planets of the Solar System, most extrasolar planets are expected to have an intrinsic, internally generated magnetic field. These magnetic fields are believed to have influence on a number of physical processes (from planetary migration to planetary mass loss, see e.g. Grießmeier [2015] and references therein), so that planets with and without magnetic fields may not behave and evolve the same way. Implications for the planets are manifold. For this reason, a number of methods have been suggested with which such a field could be detected. These methods are compared in Grießmeier [2015]. Almost all of the methods suggested so far allow for false positives, i.e. signals which could erroneously be interpreted as the signature of a planetary magnetic field. or example, chromospheric emission on a planetary host star can be triggered by a close-in planet even if this planet is unmagnetized [Preusse et al., 2006; Kopp et al., 2011]. As a second example, discrete auroras would constitute a sign of magnetic fields, but typical observations will only yield the disk-integrated signal, which are more sensitive to diffuse auroras. Such diffuse auroras have been detected over unmagnetized regions of Mars [Schneider et al., 2015], and are expected to be difficult to use as an indicator for exoplanetary magnetic fields [Grießmeier, 2015]. As a third example, we can mention the observation of an 'early ingress' during a planetary transit. It has been suggested that this would be a typical signature of a bow-shock, which would require a planetary magnetic field. However, numerical simulations [Turner et al. 2016] have shown that the signature caused by a bow 
shock would be many orders of magnitude weaker than required for this interpretation. Also, it was shown that the observed feature could be explained without a planetary magnetic field [Grießmeier, 2015, and references therein]. More examples are given in Grießmeier [2015]. For none of them can the observable be attributed unambiguously to a planetary magnetic field. The notable exception to this is the observation of exoplanetary radio emission, for which false positives can be excluded. As an added benefit, this method goes beyond the qualitative detection, and would allow to quantify the magnetic field value via the cutoff frequency of the radio emission. The detection of exoplanetary radio emission thus remains the best hope for the observation and study of exoplanetary magnetic fields [Grießmeier, 2015].

\subsection{On the energy input}

Despite the intensity of Jupiter's radio emission, the detection of an equivalent emission from an extrasolar twin of Jupiter would be challenging. The increase of distance by 5 orders of magnitude directly translates into a decrease in signal intensity by 10 orders of magnitude! With the sensitivity of current radio telescopes, this would limit the potential detection space to a sphere of $\sim 1 \mathrm{pc}$ around the solar system, with a rather limited number of targets.

As has been stated on multiple occasions and in a number of articles [see e.g. Grießmeier, 2015 for an overview], theoretical arguments indicate that the radio emission of an exoplanet could be much stronger than Jupiter's emission, giving access to a much larger number of targets for observations.

From the solar system, we know that the emitted radio power is proportional to the power input into the planetary magnetosphere. However, the precise nature of this input power is ill determined:

$$
P_{\mathrm{emi}}=\alpha P_{\mathrm{sw}, \mathrm{kin}}+\beta P_{\mathrm{sw}, \mathrm{mag}}
$$

where $P_{\text {emi }}$ is the emitted radio power, $P_{\mathrm{Sw}}$,kin is the kinetic power of the solar wind received by the planetary magnetosphere, $P_{\mathrm{Sw}}$, mag is the magnetic power received by the planetary magnetosphere, and $\alpha$ and $\beta$ are conversion efficiencies. So far we have been unable to distinguish exactly which power was dominating the power input. Usually, it was either assumed that $\alpha=0$ (i.e. the radio emission was driven by the magnetic power), or $\beta=0$ (i.e. the radio emission was driven by the kinetic power). For the solar system planets, the radial dependence of $P_{\mathrm{Sw}}$,kin and $P_{\mathrm{Sw}}$,mag is identical, so that it was not possible to distinguish between both approaches [Zarka et al., 2001; Zarka, 2007; Grießmeier et al., 2007b]. Even a mixed case $(\alpha \neq 0$ and $\beta \neq 0)$ could not be ruled out.

Recent solar system observations finally allowed to break this degeneracy. It has been shown that, similarly to Io, the satellite Ganymede generates radio emission at Jupiter. In this case, the kinetic and magnetic energy of the ambient plasma are not those of the solar wind, but of Jupiter's magnetospheric plasma. A detailed study [Zarka et al., 2017] has shown that the emitted radio power follows the same proportionality with the incoming magnetic power as the solar system planets. At the same time, the measured power is not compatible with the kinetic power scenario. Thus, we now know that $\alpha \sim 0$. More 
details are given elsewhere in this volume [Zarka et al., 2017]. This finally solves a longstanding question, allows more accurate predictions for exoplanets, and will guide the target selection for future observations. As a first step, this will be taken into account for the predictions in Section 3.

\subsection{On possible saturation}

The proportionality between (stellar wind) input power and (radio) output power is analyzed by Nichols and Milan [2016]. In the case of hot Jupiters, the radio emission is assumed to be either generated by Alfvén waves or via magnetic reconnection (in a Dungeycycle-like interaction). If it is magnetic reconnection that drives the radio emission, they find that radio emission of close-in planets may be lower than previously assumed: At small orbital distances, the magnetospheric convection may saturate (i.e. magnetospheric convection may be unable to dissipate the full available incident Poynting flux), so that the scaling laws based on this proportionality considerably overestimate the radio power for hot Jupiter. In some cases, Nichols and Milan [2016] find values lower than previously estimated by up to two orders of magnitude! On the other hand, they suggest that the power may be larger than previously assumed for planets at larger orbital distances.

\subsection{On observability}

If the intensity of exoplanetary radio emission depends on the input energy via the stellar wind, as is assumed e.g. in Equation (1), it seems obvious that the intensity is highest when the input power is highest. One way to obtain very high input power is to study planets that are very close to their host star, e.g. hot Jupiters. For this reason, hot Jupiters have long been considered to be the prime targets for observational studies [e.g. Zarka, 2007; Grießmeier et al., 2007b].

This view has already been challenged by Nichols [2011; 2012] who showed that furtherorbiting, fast-rotating, massive planets could produce intense radio emission, if they have sufficiently strong plasma sources (e.g. a moon around the planet). As discussed in Section 2.2 above, Nichols and Milan [2016] have furthermore shown that magnetospheric convection may break down for hot Jupiters.

Recent work points to an additional problem: For hot Jupiters, the outer layers of the planetary atmosphere are strongly heated by the stellar XUV flux, leading to a considerably extended planetary exosphere. At the same time, this layer becomes strongly ionized. With a high density of free electrons, this extended exosphere has a relatively high plasma frequency, which can, in certain cases, exceed the cyclotron frequency. In that case, the cyclotron maser instability (CMI), the cause for planetary radio emission, cannot operate. Worse still, even if the CMI remains possible in a small zone close to the planet, the outer layers are opaque for this radiation, which thus remains effectively trapped. Under those conditions, emission cannot escape, and no detection is possible. More details are given elsewhere in this volume [Weber et al., 2017a]. 
Whether the plasma frequency of the planetary exosphere is indeed above the frequency of the expected radio emission depends on a number of factors, including the stellar XUV activity (which, in turn, depends on the stellar mass and the stellar age), the planetary orbital distance, the planetary mass and the planetary magnetic field. The importance of this effect has to be checked on a case-by-case basis, and parametric studies are ongoing [Weber et al,. 2017b]. In any case,this is an important result, as certain hot Jupiters may not be as good targets as has been assumed previously.

\subsection{On planets with moons}

Not all studies rely on the power provided to the magnetosphere by the stellar wind. Rapidly rotating planets with strong internal plasma sources can also produce radio emission at detectable levels at orbital distances of several AU from their host star [Nichols, 2011; 2012]. The plasma source could (for example) be a moon orbiting the planet. In that case, a unipolar inductor forms, with the circuit closing well behind the moon.

Noyola et al. [2014] suggest a similar case where exoplanetary radio emission is driven by internal plasma provided by a moon. In their case, the electric current directly couples the moon to the giant planet's poles. The associated predicted fluxes are, however, rather low, well below the detection threshold of current instruments. In a follow-up study [Noyola et al., 2016] the authors extend their analysis to the case of a planet having several moons. The suggested periodic signal may, however, be difficult to detect in practice.

\subsection{On stellar magnetic fields}

All theoretical studies of exoplanetary auroral radio emission agree on the fact that the precise knowledge of the stellar wind is important, and that a fast or dense stellar wind could strongly enhance the emission [Zarka et al., 1997]. Starting from simple models (a steady stellar wind with a distance-independent velocity), theories have been refined to take into account the stellar age [Grießmeier et al., 2005; Stevens et al., 2005], stellar CMEs [Grießmeier et al., 2006b; 2007a], the planetary orbital distance [Grießmeier et al., 2007a], and the stellar rotation [Grießmeier et al., 2007a].

The next step was to take into account the observed stellar surface magnetic field (obtained with Zeeman Doppler Imaging). Using numerical models, the stellar field is extrapolated to the planetary location, giving a better handle on the planetary environment. This naturally resulted in time variability of the expected planetary radio signal with the stellar rotation and with the stellar activity cycle [Fares et al., 2010; Vidotto et al., 2012; See et al., 2015; Vidotto et al., 2015]. Numerical models also allowed to study the effect of time variability in the stellar wind [Alvarado-Gómez et al., 2016] and the dependence on planetary orbital distance [Tilley et al., 2016]. A consistent picture is yet to emerge: The different models do not necessarily use the same assumptions (e.g. radio emission caused by either the kinetic or magnetic power of the stellar wind), which makes a comparison of the different results difficult. In order to guide observational campaigns, however, it is essential to estimate radio fluxes of different planets with exactly the same method. 
Nevertheless, such simulations are a promising way to obtain more realistic insights into possible conditions in the planetary environment, and may well guide future observational campaigns.

\subsection{On exotic targets}

While most studies concentrate on radio emission from main sequence stars of $\mathrm{F}, \mathrm{G}, \mathrm{K}$ and M type, more exotic targets have already been considered in the past. As latest example, Katarzyński et al. [2016] have studied the case of planets around A stars, considering both the kinetic power scenario (see Section 2.2) and the case where the power is provided by a moon (based on the scenario by Noyola et al. [2014] mentioned in Section 2.5 above). The young stellar and planetary age are likely to result in a strong planetary magnetic field, which is favorable for radio detection.

Finally, Fujii et al. [2016] have revisited the case of planets in orbit around evolved stars. Such stars have much higher mass loss rates than their main-sequence counterparts. However, previous work [Ignace et al., 2010] indicated that the stellar wind was not sufficiently ionized to lead to a strong radio emission from the planet. Fujii et al. [2016] consider that the stellar wind could be ionized by UV and X-ray photons created by the accretion of the dense stellar wind onto the planet. In that case, the winds could indeed lead to strong magnetospheric radio emission from such planets, generating a potentially detectable radio signal. However, the estimated number of targets is small.

\section{$3 \quad$ Updated radio predictions}

Theoretical models have allowed to estimate the maximum emission frequency and the expected radio flux for known extrasolar planets. Different models have been suggested and discussed in the literature. In the following, we will take into account some of the results described above to produce updated radio predictions. We will follow the approach of Grießmeier et al. [2007b; 2011], with the following modifications:

- We have added (many!) exoplanets discovered since the last version of this table,

- we have updated the planetary and stellar parameters of the planets that were already known (esp. planetary masses and radii, and stellar ages),

- instead of comparing the radio fluxes for different models, we only use the magnetic energy model (see Section 2.2 and Zarka et al. [2017]).

- we indicate the planetary orbital distance, which may be important in the light of recent work (see Sections 2.3 - 2.4, Nichols and Milan [2016] and Weber et al. [2017a,b]),

Everything else is applied similarly to Grießmeier et al. [2007b; 2011]. In particular, we compare two different hypotheses concerning the planetary magnetic field: (a) We first 
assume that the planetary magnetic field is independent of planetary rotation (as was suggested e.g. by Reiners and Christensen [2010]; we do not, however, take the agedependence of the planetary magnetic moment into account). (b) We then assume that planetary rotation does have an influence on the planetary magnetic moment (as was done e.g. by Grießmeier et al. [2007b]), but that the planet is not tidally locked due to its orbital distance. (Note that this gives the same result as (a), but under different conditions). (c) We finally assume that the low rotation induced by tidal locking of close-in planets leads to a small planetary magnetic field (as was done e.g. by Grießmeier et al. [2007b]). Depending on the orbital distance of the planet, two or three of these hypotheses are explored: (a) and (c) for close-in planets, (a), (b) and (c) for planets at intermediate orbital distances, and (a) and (b) for planets at large orbital distances.

Table 1 shows the maximum emission frequency and the expected radio flux resulting from this estimation for a selected number of targets. Column 1 states the planetary name, and column 2 its distance to the star at periastron (to check whether an extended ionosphere might potentially prevent escape of the radio emission, see Section 2.4 and Weber et al. [2017a,b]). Columns 3 and 4 contain the maximum emission frequency and the maximum expected radio flux density at Earth for the case of a rotation-independent planetary magnetic moment. Columns 5 and 6 give the results for the case where the planetary magnetic moment depends on the planetary rotation, but where the planet is not tidally locked. Columns 7 and 8 give the results for the case where the planetary magnetic moment depends on the planetary rotation and where the planet is tidally locked. Table 1 is sorted by decreasing values in column 4 (i.e. radio flux under the hypothesis that rotation has no influence on the planetary magnetic moment). When the maximum emission frequency lies below $10 \mathrm{MHz}$, an Earth-based observation is not possible due to ionospheric cutoff. The full table is available electronically at the CDS (ftp://cdsarc.u-strasbg.fr/pub/cats/VI/151/), sorted alphabetically by column 1 in that case.

A few planets of Table 1 deserve a short discussion:

- HD 41004B b, HD 179949 b, Tau Bootes b and Ups Andromedae b have long been considered to be among the best candidates. Their predicted radio flux is still favorable; however, new planets have been detected, some of which are even more promising targets for observations.

- The flux estimation for WASP-18 b is higher than previously. The reason for this change is that the age of the system has been re-evaluated. As young stellar system (600 Myr), the star probably have a strong stellar wind, which can lead to strong planetary radio emission.

- The high flux prediction for CVSO $30 \mathrm{~b}$ is caused by the young stellar age and the very small orbital distance (0.00838 AU). Note, however, that Yu et al. [2015] put some doubt on the planetary hypothesis.

- Our approach is favorable to close-in planets. Among the more distant planets, HD 20782b and Eps Eridani are the most promising targets. 
Table 1: Expected radio emission frequencies and flux densities for a few selected targets. (The full table is available in electronic format at the CDS (ftp://cdsarc.ustrasbg.fr/pub/cats/VI/151/). Column 1: Planet name. Column 2: periastron distance. Column 3: expected maximum emission frequency (under the hypothesis that planetary rotation does not have any influence on the planetary magnetic field). Column 4: maximum expected radio flux density at Earth (under the hypothesis that planetary rotation does not have any influence on the planetary magnetic field). Column 5: expected maximum emission frequency (under the hypothesis that planetary rotation has a strong influence on the planetary magnetic field, but the planet is not tidally locked). Column 6: maximum expected radio flux density at Earth (under the hypothesis that planetary rotation has a strong influence on the planetary magnetic field, but the planet is not tidally locked). Column 7: expected maximum emission frequency (under the hypothesis that planetary rotation has a strong influence on the planetary magnetic field, and the planet is tidally locked). Column 8: maximum expected radio flux density at Earth (under the hypothesis that planetary rotation has a strong influence on the planetary magnetic field, and the planet is tidally locked). Numbers in brackets (not in the electronic table): Emission frequency is below ionospheric cutoff of Earth (emission not observable).

\begin{tabular}{|l||c||c|c||c|c||c|c|}
\hline Planet & $\begin{array}{c}r_{\min } \\
{[\mathrm{AU}]}\end{array}$ & $\begin{array}{c}f_{\mathrm{c}}^{\text {norot }} \\
{[\mathrm{MHz}]}\end{array}$ & $\begin{array}{c}\Phi_{\mathrm{max}}^{\text {norot }} \\
{[\mathrm{mJy}]}\end{array}$ & $\begin{array}{c}f_{\mathrm{c}}^{\text {rot,unlocked }} \\
{[\mathrm{MHz}]}\end{array}$ & $\begin{array}{c}\Phi_{\mathrm{max}}^{\text {rot,unlocked }} \\
{[\mathrm{mJy}]}\end{array}$ & $\begin{array}{c}f_{\mathrm{c}}^{\text {rot,locked }} \\
{[\mathrm{MHz}]}\end{array}$ & $\begin{array}{c}\Phi_{\max }^{\text {rot,locked }} \\
{[\mathrm{mJy}]}\end{array}$ \\
\hline \hline GJ 1214 b & 0.010 & 16 & 4500 & - & - & 6.0 & 6300 \\
HD 41004 B b & 0.017 & 140 & 910 & - & - & 55 & 1200 \\
CVSO 30 b & 0.008 & 30 & 790 & - & - & 28 & 810 \\
GJ 436 b & 0.023 & 18 & 780 & - & - & 4.5 & 1200 \\
HD 189733 b & 0.031 & 21 & 520 & - & - & 6.0 & 800 \\
Kepler-42 c & 0.006 & 41 & 280 & - & - & 38 & 280 \\
GJ 1132 b & 0.015 & 23 & 190 & - & - & 8.0 & 260 \\
tau Boo b & 0.042 & 74 & 170 & - & - & 15 & 290 \\
55 Cnc e & 0.015 & 30 & 150 & - & - & 19 & 170 \\
WASP-18 b & 0.020 & 92 & 140 & - & - & 46 & 180 \\
HD 73256 b & 0.036 & 34 & 100 & - & - & 8.2 & 160 \\
ups And b & 0.058 & 14 & 75 & - & - & 2.2 & 140 \\
HD 179949 b & 0.045 & 20 & 71 & - & - & 4.3 & 120 \\
WASP-33 b & 0.026 & 14 & 63 & - & - & 5.7 & 85 \\
HD 20782 b & 0.041 & 38 & 40 & - & - & 0.2 & 250 \\
HD 102195 b & 0.049 & 11 & 37 & - & - & 2.0 & 65 \\
HATS-24 b & 0.019 & 22 & 18 & - & - & 8.2 & 25 \\
eps Eridani b & 1.01 & 53 & 2.4 & 53 & 2.4 & - & - \\
55 Cnc b & 0.112 & 20 & 2.9 & 20 & 2.9 & 3.3 & 5.3 \\
V830 Tau b & 0.057 & 18 & 0.3 & - & - & 2.8 & 0.6 \\
\hline
\end{tabular}




\section{Observational studies}

While most radio observations of extrasolar planets started after the publication of the first theoretical studies, a few observational campaigns took place way before theoretical studies tried to estimate fluxes or emission frequencies for extrasolar planets. The first observational campaigns (the earliest going back at least to 1977) took place even before the discovery of extrasolar planets [Yantis et al., 1977]!

Table 2 gives an update of the list of observations presented in Grießmeier et al. [2006a] and shows all currently published attempts at detecting exoplanetary radio emission, sorted by telescope and by observing frequency. The observed frequency range covers three orders of magnitude, spanning from below $10 \mathrm{MHz}$ to almost $10 \mathrm{GHz}$.

One can see that, compared to our predictions (Section 3), not all observational attempts took place in the most favorable frequency range. If our predictions can be believed, the most appropriate instruments for exoplanetary radio studies are UTR-2, LOFAR, and the VLA, with smaller chances for MWA and GMRT.

A few observations have claimed tentative detections [Lecavelier des Etangs et al., 2013; Sirothia et al., 2014], none of which has been confirmed to date. Among the most recent observations, those by Bower et al. [2016] led to a tentative detection. However, this signal was probably produced by the host star [Bower et al., 2016]. Thus, so far no firm detection has been achieved.

As can be seen, the sensitivity of modern radio telescopes is very close to the radio fluxes predicted by theory (see e.g. Section 3). In particular, the predicted flux for the 15 most favorable planets of Table 1 is above the theoretical upper limit of $21 \mathrm{mJy}$ of the LOFAR low-frequency band [Turner et al., 2017].

With instrument sensitivities close to the theoretical predictions, more observations (and the re-analysis of previous observations) are currently ongoing. One example of an ongoing observational campaign is described elsewhere in this volume [Turner et al., 2017].

\section{Conclusions}

Auroral radio emission from extrasolar planets remains an active field of research. Recent theoretical studies include effects that have so far been neglected, and some consider "exotic" targets which have not been much looked into in the past. We have reviewed the most recent theoretical studies. A few of these studies have the potential to change the way targets are selected for observational campaigns. In particular, (1) the "kinetic energy" model can be discarded, while the "magnetic energy" scenario is confirmed by solar system data (Section 2.2), (2) for hot Jupiters, it is possible that the radio emission saturates (Section 2.3), (3) still for hot Jupiters, the emission may, in certain cases, be trapped, leading to the absence of a detectable signal (Section 2.4).

We have also presented an up-to-date list of observational campaigns, and an updated list of predicted radio fluxes and emission frequencies obtained from simple models. 
Table 2: Published detection attempts, sorted by instrument and frequency. *: see text.

\begin{tabular}{|c|c|c|c|}
\hline Telescope & Frequency & Sensitivity & Reference \\
\hline UTR-2 & $\begin{array}{l}7-35 \mathrm{MHz} \\
18-32 \mathrm{MHz} \\
12-32 \mathrm{MHz}\end{array}$ & $\begin{array}{c}2000-4000 \mathrm{mJy} \\
100-1600 \mathrm{mJy} \\
\sim 100 \mathrm{mJy}\end{array}$ & $\begin{array}{l}\text { Zarka et al. }[1997] \\
\text { Ryabov et al. }[2004] \\
\text { Zarka et al. }[2011]\end{array}$ \\
\hline Clark Lake & $26.3 \mathrm{MHz}$ & $1000 \mathrm{mJy}$ & Yantis et al. [1977] \\
\hline LOFAR & $10-60 \mathrm{MHz}$ & $21 \mathrm{mJy}$ & Turner et al. [2017] \\
\hline MWA & $\begin{array}{l}154 \mathrm{MHz} \\
154 \mathrm{MHz}\end{array}$ & $\begin{array}{l}3-50 \mathrm{mJy} \\
4-235 \mathrm{mJy}\end{array}$ & $\begin{array}{l}\text { Murphy et al. [2015] } \\
\text { Lynch et al. }[2017]\end{array}$ \\
\hline GMRT & $\begin{array}{l}153 \mathrm{MHz} \\
157 \mathrm{MHz} \\
148 \mathrm{MHz} \\
150 \mathrm{MHz} \\
153 \mathrm{MHz} \\
150 \mathrm{MHz} \\
244 \mathrm{MHz} \\
614 \mathrm{MHz}\end{array}$ & $\begin{array}{c}2 \mathrm{mJy} \\
\\
7.8-15.5 \mathrm{mJy} \\
2.1-3.6 \mathrm{mJy} \\
3.9 \mathrm{mJy} \\
1.2 \mathrm{mJy} \\
8.7-136 \mathrm{mJy} \\
2 \mathrm{mJy} \\
0.16 \mathrm{mJy}\end{array}$ & $\begin{array}{l}\text { Majid et al. [2006]; } \\
\text { Winterhalter et al. [2006] } \\
\text { George and Stevens [2007] } \\
\text { Lecavelier des Etangs et al. [2011] } \\
\text { Lecavelier des Etangs et al. [2013] } \\
\text { Hallinan et al. [2013] } \\
\text { Sirothia et al. [2014] } \\
\text { Lecavelier des Etangs et al. [2009] } \\
\text { Lecavelier des Etangs et al. [2009] }\end{array}$ \\
\hline GBT & $307-347 \mathrm{MHz}$ & $81 \mathrm{mJy}$ & Smith et al. $[2009]$ \\
\hline VLA & $\begin{array}{l}74 \mathrm{MHz} \\
74 \mathrm{MHz} \\
74 \mathrm{MHz} \\
74 \mathrm{MHz} \\
74 \mathrm{MHz} \\
330 \mathrm{MHz} \\
333 \mathrm{MHz} \\
325 \mathrm{MHz} \\
1460 \mathrm{MHz} \\
1465 \mathrm{MHz} \\
1425 \mathrm{MHz} \\
4900 \mathrm{MHz} \\
6000 \mathrm{MHz}\end{array}$ & $\begin{array}{c}50 \mathrm{mJy} \\
120 \mathrm{mJy} \\
300 \mathrm{mJy} \\
135-300 \mathrm{mJy} \\
10-33 \mathrm{mJy} \\
30 \mathrm{mJy} \\
1-10 \mathrm{mJy} \\
1.7 \mathrm{mJy} \\
0.3 \mathrm{mJy} \\
0.02-0.07 \mathrm{mJy} \\
0.048 \mathrm{mJy} \\
0.15 \mathrm{mJy} \\
0.9 \mathrm{mJy}^{\star}\end{array}$ & $\begin{array}{l}\text { Bastian et al. [2000] } \\
\text { Farrell et al. [2003] } \\
\text { Lazio et al. [2004] } \\
\text { Lazio and Farrell [2007] } \\
\text { Lazio et al. [2010a] } \\
\text { Winglee et al. [1986] } \\
\text { Bastian et al. [2000] } \\
\text { Lazio et al. [2010b] } \\
\text { Winglee et al. [1986] } \\
\text { Bastian et al. [2000] } \\
\text { Lazio et al. [2010b] } \\
\text { Winglee et al. [1986] } \\
\text { Bower et al. [2016] }\end{array}$ \\
\hline VLBA & $8400 \mathrm{MHz}$ & $0.5 \mathrm{mJy}^{\star}$ & Bower et al. [2016] \\
\hline WSRT & $1700 \mathrm{MHz}$ & $0.042 \mathrm{mJy}$ & Stroe et al. $[2012]$ \\
\hline Effelsberg & $4850 \mathrm{MHz}$ & $0.8 \mathrm{mJy}$ & Guenther [priv. comm.] \\
\hline Mizusawa & $8600 \mathrm{MHz}$ & $1000 \mathrm{mJy}$ & Shiratori et al. [2006] \\
\hline
\end{tabular}

A lot of progress has been made since the first PRE proceedings article on radio emission from extrasolar planets. Still more work remains to be done, including, of course, a first firm detection! Future PRE conferences and proceedings will contribute to this ongoing story. 
Acknowledgments. We thank J. Schneider for providing data via "The extrasolar planet encyclopedia" (http://exoplanet.eu/), fully described in Schneider et al. [2011]. The Editors thank Luca Fossati and one anonymous reviewer for their help in evaluating this paper.

\section{References}

Alvarado-Gómez, J. D., G. A. J. Hussain, O. Cohen, J. J. Drake, C. Garraffo, J. Grunhut, and T. I. Gombosi, Simulating the environment around planet-hosting stars II. Stellar winds and inner astrospheres, Astron. Astrophys., 594, A95, 2016.

Bastian, T.S., G. A. Dulk, and Y. Leblanc, A search for radio emission from extrasolar planets, Astrophys. J., 545, 1058-1063, 2000.

Bower, G. C., L. Loinard, S Dzib, P. A. B. Galli, G. N. Ortiz-León, C. Moutou, and J.F. Donati, Variable radio emission from the young stellar host of a hot Jupiter, Astrophys. J., 830, id.107, 5 pp., 2016.

Fares, R., J.-F. Donati, C. Moutou, M. M. Jardine, J.-M. Grießmeier, P. Zarka, E. L. Shkolnik, D. Bohlender, C. Catala, and A. C. Cameron, Searching for star-planet interactions within the magnetosphere of HD189733, MNRAS, 406, 409-419, 2010.

Farrell, W. M., T. J. Lazio, M. D. Desch, T. Bastian, and P. Zarka, Limits on the magnetosphere/stellar wind interactions for the extrasolar planet about Tau Bootes, in Scientific Frontiers in Research on Extrasolar Planets, edited by D. Deming, and S. Seager, ASP Conference Series, 294, 151-156, 2003.

Fujii, Y., D. S. Spiegel, T. Mroczkowski, J. Nordhaus, N. T. Zimmerman, A. R. Parsons, M. Mirbabayi, and N. Madhusudhan, Radio emission from red-giant hot Jupiters, Astron. Astrophys., 820, id.122, 15 pp., 2016.

George, S. J., and I. R. Stevens, Giant Metrewave Radio Telescope low-frequency observations of extrasolar planetary systems, Mon. Not. Roy. Astron. Soc., 382, 455-460, 2007.

Grießmeier, J.-M., Detection methods and relevance of exoplanetary magnetic fields, in Characterizing stellar and exoplanetary environments, Astrophysics and Space Science Library, 411, edited by H. Lammer and M. Khodachenko, Springer, Switzerland, 213-237, 2015.

Grießmeier, J.-M., U. Motschmann, G. Mann, and H. O. Rucker, The influence of stellar wind conditions on the detectability of planetary radio emissions, Astron. Astrophys., 437, 717-726, 2005.

Grießmeier, J.-M., U. Motschmann, K.-H. Glassmeier, G. Mann and H. O. Rucker, The potential of exoplanetary radio emissions as an observation method, in Tenth Anniversary of 51 Peg-b: Status of and Prospects for hot Jupiter studies, edited by L. Arnold, F. Bouchy, and C. Moutou, Platypus Press, 259-266, 2006a. 
Grießmeier, J.-M., U. Motschmann, M. Khodachenko, and H. O. Rucker, The influence of stellar Coronal Mass Ejections on exoplanetary radio emission, in Planetary Radio Emissions VI, edited by H. O. Rucker, W. S. Kurth, and G. Mann, Austrian Academy of Sciences Press, Vienna, 571-580, 2006b.

Grießmeier, J.-M., S. Preusse, M. Khodachenko, U. Motschmann, G. Mann, and H. O. Rucker, Exoplanetary radio emission under different stellar wind conditions, Planet. Space Sci., 55, 618-630, 2007a.

Grießmeier, J.-M., P. Zarka and H. Spreeuw, Predicting low-frequency radio fluxes of known extrasolar planets, Astron. Astrophys., 475, 359-368, 2007b.

Grießmeier, J.-M., P. Zarka, and J. N. Girard, Observation of planetary radio emissions using large arrays, Radio Sci., 46, RS0F09, 2011.

Hallinan, G., S. K. Sirothia, A. Antonova, C. H. Ishwara-Chandra, S. Bourke, J. G. Doyle, J. Hartman, and A. Golden, Looking for a pulse: A search for rotationally modulated radio emission from the Hot Jupiter, $\tau$ Boötis b, Astrophys. J., 762, id.34, 4 pp., 2013.

Ignace, R., M. L. Giroux and D. G. Luttermoser, Radio emissions from substellar companions of evolved cool stars, MNRAS, 402, 2609-2616, 2010.

Katarzyński, K., M. Gawrónski, and K. Goździewski, Search for exoplanets and brown dwarfs with VLBI, MNRAS, 461, 929-938, 2016.

Kopp, A., S. Schilp, and S. Preusse, Magnetohydrodynamic simulations of the magnetic interaction of hot Jupiters with their host stars: A numerical experiment, Astrophys. J., 729, id.116, 8 pp., 2011.

Lazio, T. J. W., and W. M. Farrell, Magnetospheric emissions from the planet orbiting $\tau$ Bootis: A multi-epoch search, Astrophys. J., 668, 1182-1188, 2007.

Lazio, T. J. W., W. M. Farrell, J. Dietrick, E. Greenlees, E. Hogan, C. Jones, and L. A. Hennig, The radiometric Bode's law and extrasolar planets, Astrophys. J., 612, 511-518, 2004 .

Lazio, T. J. W., S. Carmichael, J. Clark, E. Elkins, P. Gudmundsen, Z. Mott, M. Szwajkowski, and L. A. Hennig, A blind search for magnetospheric emissions from planetary companions to nearby solar-type stars, Astron. J., 139, 96-101, 2010.

Lazio, T. J. W., P. D. Shankland, W. M. Farrell, and D. L. Blank, Radio observations of HD 80606 near planetary periastron, Astron. J., 140, 1929-1933, 2010.

Lecavelier des Etangs, A., S. K. Sirothia, Gopal-Krishna, and P. Zarka, GMRT radio observations of the transiting extrasolar planet HD 189733 b at 244 and $614 \mathrm{MHz}$, Astron. Astrophys., 500, L51-L54, 2009.

Lecavelier des Etangs, A., S. K. Sirothia, Gopal-Krishna, and P. Zarka, GMRT search for $150 \mathrm{MHz}$ radio emission from the transiting extrasolar planets HD189733 b and HD209458 b, Astron. Astrophys., 533, A50, 2011. 
Lecavelier des Etangs, A., S. K. Sirothia, Gopal-Krishna, and P. Zarka, Hint of $150 \mathrm{MHz}$ radio emission from the Neptune-mass extrasolar transiting planet HAT-P-11b, Astron. Astrophys., 552, A65, 2013.

Lynch, C. R., T. Murphy, D. L. Kaplan, M. Ireland, and M. E. Bell, A search for circularly polarized emission from young exoplanets, MNRAS, 467, 3447-3453, 2017.

Majid, W., D. Winterhalter, I. Chandra, T. Kuiper, J. Lazio, C. Naudet, and P. Zarka, Search for radio emission from extrasolar planets: Preliminary analysis of GMRT data, in Planetary Radio Emissions VI, edited by H. O. Rucker, W. S. Kurth, and G. Mann, Austrian Academy of Sciences Press, Vienna, 589-594, 2006.

Murphy, T., M. E. Bell, D. L. Kaplan, B. M. Gaensler, A. R. Offringa, E. Lenc, N. HurleyWalker, G. Bernardi, J. D. Bowman, F. Briggs, R. J. Cappallo, B. E. Corey, A. A. Deshpande, D. Emrich, R. Goeke, L. J. Greenhill, B. J. Hazelton, J. N. Hewitt, M. JohnstonHollitt, J. C. Kasper, E. Kratzenberg, C. J. Lonsdale, M. J. Lynch, S. R. McWhirter, D. A. Mitchell, M. F. Morales, E. Morgan, D. Oberoi, S. M. Ord, T. Prabu, A. E. E. Rogers, D. A. Roshi, N. Udaya Shankar, K. S. Srivani, R. Subrahmanyan, S. J. Tingay, M. Waterson, R. B. Wayth, R. L. Webster, A.R. Whitney, A. Williams, and C. L. Williams, Limits on low-frequency radio emission from southern exoplanets with the Murchison Widefield Array, MNRAS, 446, 2560-2565, 2015.

Nichols, J. D., Magnetosphere-ionosphere coupling at Jupiter-like exoplanets with internal plasma sources: Implications for detectability of auroral radio emissions, MNRAS, 414, 2125-2138, 2011.

Nichols, J. D., Candidates for detecting exoplanetary radio emissions generated by magnetosphere-ionosphere coupling, MNRAS Lett., 427, L75-L79, 2012.

Nichols, J.D., and S.E. Milan, Stellar wind-magnetosphere interaction at exoplanets: Computations of auroral radio powers, MNRAS, 461, 2353-2366, 2016.

Noyola, J.P., S. Satyal, and Z.E. Musielak, Detection of exomoons through observation of radio emissions, Astrophys. J., 791, id.25, 5 pp., 2014.

Noyola, J. P., S. Satyal, and Z. E. Musielak, On the radio detection of multiple-exomoon systems due to plasma torus sharing, Astrophys. J., 821, id.97, 8 pp., 2016.

Preusse, S., A. Kopp, J. Buchner, and U. Motschmann, A magnetic communication scenario for hot Jupiters, Astron. Astrophys., 460, 317-322, 2006.

Reiners, A., and U.R. Christensen, A magnetic field evolution scenario for brown dwarfs and giant planets, Astron. Astrophys., 522, A13, 2010.

Ryabov, V.B., P. Zarka, and B.P. Ryabov, Search of exoplanetary radio signals in the presence of strong interference: Enhancing sensitivity by data accumulation, Planet. Space Sci., 52, 1479-1491, 2004.

Schneider, J., C. Dedieu, P. Le Sidaner, R. Savalle, and I. Zolotukhin, Defining and cataloging exoplanets: The exoplanet.eu database, Astron. Astrophys., 532, A79, 2011. 
Schneider, N. M., J. I. Deighan, S. K. Jain, A. Stiepen, A. I. F. Stewart, D. Larson, D. L. Mitchell, C. Mazelle, C. O. Lee, R. J. Lillis, J. S. Evans, D. Brain, M. H. Stevens, W. E. McClintock, M. S. Chaffin, M. Crismani, G. M. Holsclaw, F. Lefevre, D. Y. Lo, J. T. Clarke, F. Montmessin, and B. M. Jakosky, Discovery of diffuse aurora on Mars, Science, 350, id.0313, 2015.

See, V., M. Jardine, R. Fares, J.-F. Donati, and C. Moutou, Time-scales of close-in exoplanet radio emission variability, MNRAS, 450, 4323-4332, 2015.

Shiratori, Y., H. Yokoo, T. Sasao, O. Kameya, K. Iwadate, and K. Asari, Ten years of quests for radio bursts from extrasolar planets, in Tenth Anniversary of 51 Peg-b: Status of and Prospects for hot Jupiter studies, edited by L. Arnold, F. Bouchy, and C. Moutou, Platypus Press, 290-292, 2006.

Sirothia, S. K., A. Lecavelier des Etangs, Gopal-Krishna, N. G. Kantharia, and C. H. Ishwar-Chandra, Search for $150 \mathrm{MHz}$ radio emission from extrasolar planets in the TIFR GMRT Sky Survey, Astron. Astrophys., 562, A108, 2014.

Smith, A. M. S., A. Collier Cameron, J. Greaves, M. Jardine, G. Langston, and D. Backer, Secondary radio eclipse of the transiting planet HD 189733 b: An upper limit at 307-347 MHz, MNRAS, 395, 335-341, 2009.

Stevens, I. R., Magnetospheric radio emission from extrasolar giant planets: The role of the host stars, MNRAS, 356, 1053-1063, 2005.

Stroe, A., I. A. G. Snellen, and H. J. A. Röttgering, A stringent upper limit to $18 \mathrm{~cm}$ radio emission from the extrasolar planet system $\tau$ Boötis, Astron. Astrophys., 546, A116, 2012 .

Tilley, M. A., E. M. Harnett, and R. M. Winglee, Extrasolar giant magnetospheric response to steady-state stellar wind pressure at 10, 5, 1, and 0.2 AU, Astrophys. J., 827, id. 77,15 pp., 2016.

Turner, J. D., D. Christie, P. Arras, R. E. Johnson, and C. Schmidt, Investigation of the environment around close-in transiting exoplanets using CLOUDY, MNRAS, 458, 3880-2891, 2015.

Turner, J. D., J.-M. Grießmeier, P. Zarka, and I. Vasylieva, The search for radio emission from exoplanets using LOFAR low-frequency beam-formed observations: Data pipeline and preliminary results for the 55 Cnc system, in Planetary Radio Emissions VIII, edited by G. Fischer, G. Mann, M. Panchenko, and P. Zarka, Austrian Academy of Sciences Press, Vienna, 301-313, 2017.

Vidotto, A. A., R. Fares, M. M. Jardine, J.-F. Donati, M. Opher, V. Jatenco-Pereira, and T. I. Gombosi, M. Opher, C. Moutou, C. Catala, and T. I. Gombosi, The stellar wind cycles and planetary radio emission of the $\tau$ Boo system, MNRAS, 423, 3285-3298, 2012 .

Vidotto, A. A., R. Fares, M. Jardine, C. Moutou, and J.-F. Donati, On the environment surrounding close-in exoplanets MNRAS, 449, 4117-4130, 2015. 
Weber, C., H. Lammer, I.-F. Shaikhislamov, J.-M. Chadney, N. Erkaev, M. L. Khodachenko, J.-M. Grießmeier, H. O. Rucker, C. Vocks, W. Macher, P. Odert and K.-G. Kislyakova, On the Cyclotron Maser Instability in magnetospheres of Hot Jupiters-Influence of ionosphere models, in Planetary Radio Emissions VIII, edited by G. Fischer, G. Mann, M. Panchenko, and P. Zarka, Austrian Academy of Sciences Press, Vienna, 317-329, 2017a.

Weber, C., H. Lammer, I. F. Shaikhislamov, J. M. Chadney, M. L. Khodachenko, J.M. Grießmeier, H. O. Rucker, C. Vocks, W. Macher, P. Odert and K. G. Kislyakova, How expanded ionospheres of Hot Jupiters can prevent escape of radio emission generated by the Cyclotron Maser Instability, MNRAS, 469, 3, 3505-3517, 2017b.

Winglee, R. M., G. A. Dulk, and T.S. Bastian, A search for cyclotron maser radiation from substellar and planet-like companions of nearby stars, Astrophys. J. Lett., 309, L59-L62, 1986.

Winterhalter, D., T. Kuiper, W. Majid, I. Chandra, J. Lazio, P. Zarka, C. Naudet, G. Bryden, W. Gonzalez, and R. Treumann, Search for radio emissions from extrasolar planets: The observation campaign, in Planetary Radio Emissions VI, edited by H. O. Rucker, W.S. Kurth, and G. Mann, Austrian Academy of Sciences Press, Vienna, 595-602, 2006.

Yantis, W.F., W.T. Sullivan, III, and W. C. Erickson, A search for extra-solar Jovian planets by radio techniques, Bull. American Astron. Soc., 9, p.453, 1977.

$\mathrm{Yu}$, L., et al. (19 co-authors), Tests of the planetary hypothesis for PTFO 8-8695b, Astrophys. J., 812, id.48, 18 pp., 2015.

Zarka, P., Plasma interactions of exoplanets with their parent star and associated radio emissions, Planet. Space Sci., 55, 598-617, 2007.

Zarka, P., The search for exoplanetary radio emissions, in Planetary Radio Emissions VII, edited by H. O. Rucker, W. S. Kurth, P. Louarn, and G. Fischer, Austrian Academy of Sciences Press, Vienna, 287-301, 2011.

Zarka, P., J. Queinnec, B. P. Ryabov, V. B. Ryabov, V. A. Shevchenko, A. V. Arkhipov, H. O. Rucker, L. Denis, A. Gerbault, P. Dierich, and C. Rosolen, Ground-based high sensitivity radio astronomy at decameter wavelengths, in Planetary Radio Emissions IV, edited by H. O. Rucker, S. J. Bauer, and A. Lecacheux, Austrian Academy of Sciences Press, Vienna, 101-127, 1997.

Zarka, P., R. A. Treumann, B. P. Ryabov, and V. B. Ryabov, Magnetically-driven planetary radio emissions and application to extrasolar planets, Astrophys. Space Sci., 277, 293-300, 2001.

Zarka, P., M.S. Marques, C. Louis, V.B. Ryabov, L. Lamy, E. Echer, and B. Cecconi, Radio emission from satellite-Jupiter interactions (especially Ganymede), in Planetary Radio Emissions VIII, edited by G. Fischer, G. Mann, M. Panchenko, and P. Zarka, Austrian Academy of Sciences Press, Vienna, 45-58, 2017. 
